\title{
CRY links the circadian clock and CREB-mediated gluconeogenesis
}

\author{
Megumi Hatori ${ }^{1}$, Satchidananda Panda ${ }^{1}$ \\ ${ }^{I}$ The Salk Institute for Biological Studies, 10010 North Torrey Pines Road, La Jolla, CA 92037, USA \\ Cell Research (2010) 20:1285-1288. doi:10.1038/cr.2010.152; published online 9 November 2010
}

Circadian oscillators based on a transcriptional feedback loop exist in almost all cells of animals. The cellular oscillators synchronize each other via paracrine or systemic communications, resulting in rhythmic changes of tissue- and whole body-level physiologies and behaviors. Circadian regulation of metabolism is well documented and disruption of such temporal regulation is known to predispose organisms to metabolic diseases. However, the underlying mechanism of circadian regulation of metabolism is complex and incompletely understood. The research has mainly focused on the transcriptional mechanisms by which circadian clock components regulate metabolic gene expression. A recent paper by Zhang et al. in Nature Medicine [1] for the first time elucidated a non-nuclear role of a circadian clock protein CRYPTOCHROME (CRY) in mice; CRY regulates the circadian changes of hepatic gluconeogenesis, possibly through the interaction with a G protein, Gs $\alpha$ subunit, which leads to temporal regulation of glucagon signaling triggering CREB phosphorylation (Figure 1).

The circadian oscillator is based on transcriptional mechanisms in which the BMAL1 and CLOCK (or NPAS2) transcription factor heterodimer binds

Correspondence: Satchidananda Panda Tel: +1-858-453-4100 ext 1912

E-mail: panda@salk.edu to the cis-acting E-box elements in Period (Per1-Per3) and Cryptochrome (Cryl and Cry2) genes and drive their transcription. Subsequently, PER and CRY proteins enter the nucleus and inhibit the transactivation by CLOCK-

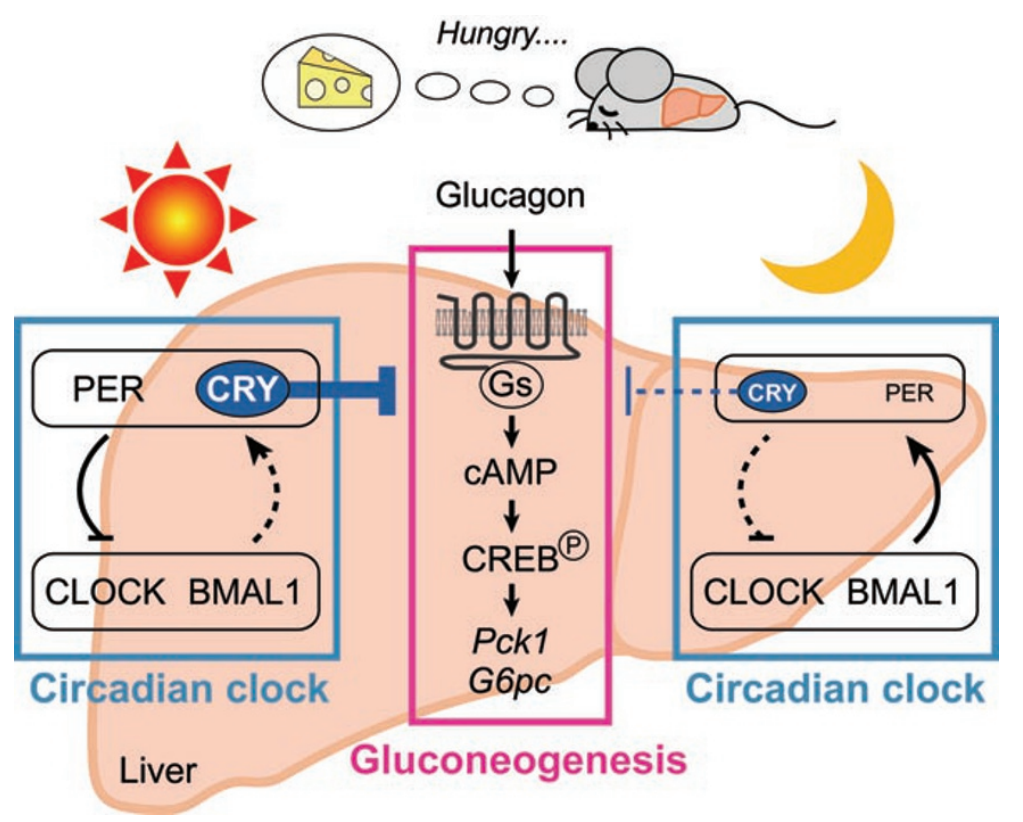

Figure 1 Circadian clock protein CRYPTOCHROME controls gluconeogenesis in the liver. The circadian core loop (blue box) is constituted of transcriptional activators CLOCK and BMAL1 and repressors PER and CRY. In the mouse liver, CLOCK-BMAL1 heterodimer activates expression of Per and Cry genes at early night (right panel). Then translated PER and CRY proteins inhibit CLOCK-BMAL1 activity in the morning (left panel) to form a negative feedback loop which cycles once a day. Fasting signal induces gluconeogenesis program through activation of CAMP/CREB signaling (red box). Zhang et al. [1] found that $C R Y$ inhibits glucagon-stimulated cAMP production possibly through interaction with a G-protein Gs a subunit. The circadian rhythm of CRY protein levels form a "gate" for the response of the gluconeogenesis program to fasting: in the morning when CRY levels are high, the response is small (left panel), while at early night when CRY levels are low and mice normally start to eat, the response is large (right panel). 
BMAL1, thus producing alternating cycles of transcriptional activation and repression. Several additional gene products including REV-ERB and ROR participate in maintaining the accuracy of this rhythm (see a review [2] for details). The circadian oscillator exists in many tissues and organs [3]. The master circadian pacemaker resident in the hypothalamic suprachiasmatic nucleus generates daily rhythms of activity-rest (and hence feeding and fasting), and the peripheral oscillator in the liver plays a major role in energy homeostasis in the face of periodic fasting and feeding.

Given the transcriptional nature of the core oscillator, it is presumed that circadian regulation of output physiology and metabolism is at least partly mediated by transcriptional oscillation of the relevant genes. Accordingly, genome-wide temporal expression profiling studies have shown that up to $15 \%$ of the protein coding genes in the liver of mice fed ad libitum show circadian rhythmic expression. Several of these rhythmic transcripts encode rate-limiting enzymes of metabolic pathways including those involved in energy homeostasis [4]. The relevance of circadian regulation to metabolism has been established by the observation that mice lacking normal CLOCK or BMAL1 function in the entire body or in specific metabolic organs show abnormal glucose homeostasis leading to metabolic diseases [5-8]. Therefore, understanding the mechanism of circadian regulation of metabolism would open novel approaches to treat the growing epidemic of metabolic diseases.

The mechanisms underlying circadian and metabolic regulation of liver function are complex and reciprocal in nature. Expression profiling of the Clock mutant liver has shown that most of the rhythmic transcripts are not directly regulated by the clock components, rather indirect mechanisms generate such rhythms [9]. Furthermore, daily transcriptional oscillation in a large number of hepatic transcripts is gener- ated by the natural cycles of feeding and fasting even in the complete absence of a clock [10]. However, the oscillations are robust in the presence of a functional oscillator, which suggests both the circadian clock and feeding and fasting regulated mechanisms synergize to generate robust transcriptional rhythms.

The groups of Drs Steve Kay and Marc Montminy with complementary expertise in the circadian clock and the metabolism focused on the dynamic regulation of hepatic gluconeogenesis. Gluconeogenesis is the process of glucose synthesis to maintain the blood glucose levels under fasting (starving) conditions. Several components of gluconeogenic pathway show circadian rhythms in transcript levels and are also known to be regulated by feeding and fasting. Disrupted gluconeogenesis is a hallmark feature of several metabolic diseases.

Gluconeogenesis is tightly controlled by multiple signaling mechanisms among which the peptide hormone glucagon is a prominent player. During fasting, glucagon secreted by the pancreas binds to a G-protein coupled receptor (GPCR) called the glucagon receptor (Figure 1) in hepatocytes. This binding activates the downstream G-protein Gs, which triggers increased intracellular cyclic AMP (cAMP) and phosphorylation of CREB (cAMP response element-binding). Transcriptionally active p-CREB drives transcription of gluconeogenic genes, such as Pckl [encoding PEPCK (phosphoenolpyruvate carboxykinase)] and G6pc (encoding G6Pase [glucose-6phosphatase]), from cis-acting CRE (cAMP response element) sites in their promoter regions.

The levels of p-CREB and a large number of CREB-regulated transcripts show rhythmic expression in the liver [10]. Zhang et al. first tested whether the glucagon-mediated activation of CREB target gene transcription shows rhythmic pattern. They intravenously delivered an adenovirus encoding CRE-driven luciferase (luc) reporter into mice. The activity of reporter in the liver can be monitored by an in vivo imaging system. Fasted mice showed high hepatic CRE-luc activity during early nighttime. In contrast, in the morning, fasted mice showed less CRE-luc activity compared to nighttime. Even exogenous injection of excess glucagon at both times led to the day-night differences in CRE-luc activity, thus ruling out rhythmic glucagon production as a potential cause. This morning dip in CRE-luc activity reflected reduced CREB transcriptional activity and coincided with similar dip in CLOCK-BMAL1 activity. Since the morning dip in CLOCK-BMAL1 activity is due to repression by peak levels of the PER-CRY complex, the authors speculated that either one or more of PER1-3 and CRY1-2 may downregulate CRE-mediated gene expression. The circadian oscillator can also regulate gene expression via non-cell autonomous systemic cues [11]. Therefore, they performed cell-based assays to test whether overexpression of clock proteins can modulate CRE-luc activity triggered by a Gs coupled-GPCR and its ligand. Among various clock components tested, overexpression of CRY1 and CRY2 inhibited CRE-luc activity, suggesting the specific roles of CRY on inhibition of gluconeogenesis in the morning.

This inhibitory effect by CRY was also observed in vivo with CRE-luc mice. Adenoviral introduction of CRY1 reduced the hepatic CRE activity of fasted mice during early nighttime, when endogenous CRY protein level is low. In accordance to the reduced CRE activity, the expression of gluconeogenic genes was also inhibited, resulting in low blood glucose concentration. In contrast to the CRY overexpression, Cryl and Cry 2 double KO mice or acute RNAi inhibition of Crys in the liver increased the CRE activity, the expression of gluconeogenic genes, and the blood glucose level of fasted mice in the morning. The inhibitory effect 
of CRY on the glucose production was also observed in $d b / d b$ mice, a mouse model of obesity and diabetes with excess gluconeogenesis. When CRY1 was adenovirally overexpressed in the liver of $d b / d b$ mice, the mice showed reduced blood glucose levels in fasted condition, thus establishing that acute manipulation of the function of a clock component can be a strategy to perturb gluconeogenesis and regulate blood sugar levels.

Which step(s) of the gluconeogenesis cascade are inhibited by CRY? From the observation that fasting- or glucagon-induced cAMP concentration fluctuates in $\sim 24 \mathrm{~h}$ rhythms with trough level coinciding with the peak level of CRY expression, the authors speculated that CRY might inhibit a step upstream of cAMP production. When mice were starving, adenoviral transduction of CRY reduced the hepatic concentration of cAMP, suggesting that the point of CRY function might be at the level of Gs, or at the level of adenylate cyclase (AC) that produces cAMP from ATP. The observation however, did not distinguish between a direct role of CRY or an unknown CRY-regulated protein in cAMP regulation. To differentiate this, the authors used a membrane preparation to measure isoproterenol activation of Gs-coupled $\beta$ adrenergic receptor leading to increased cAMP production. In this assay, membrane preps from wild-type cells mixed with CRY1-expressing cytoplasm produced less cAMP while antibody-mediated depletion of CRY increased cAMP production, thus suggesting a direct role of CRY (not of its downstream transcriptional target) in inhibiting GPCRstimulated cAMP production.

Finally, the authors tested which step in the cAMP production is inhibited by CRY. CRY failed to inhibit forskolin activation of $\mathrm{AC}$, thus ruling out $\mathrm{AC}$ as a point of CRY regulation. That left Gs as a potential point of regulation. In fact, the authors showed that CRY can bind to Gs in reciprocal binding assays.
Such a physical interaction suggests a potential role of CRY in inhibiting Gs function.

Novel findings about CRY regulation of Gs signaling leading to CREB activation is a paradigm shifting observation that has widespread repercussions. Although CRY proteins have been studied in multiple organisms, their functions have always been described as transcriptional regulators, and their interacting partners have no protein structure similarity with the Gs proteins. Hence, the observation raises a new function of CRY proteins outside the nucleus. It also raises a question whether CRY inhibition of G-protein is specific to Gs or it can modulate other G-proteins. On the other hand, the mechanism of downregulation of $\mathrm{G}$ proteins is of intense scrutiny. Typically, activated and GTP-bound G-proteins catalyze the breakdown of GTP to GDP, which leads to inactive GDP-bound G-protein. Several proteins including the Regulators of G-protein Signaling (RGS) proteins accelerate the GTPase activity of G-proteins and are thus said to be GTPase-activating proteins (GAPs) [12]. A potential mechanism by which CRY can inhibit Gs activity is being a GAP, facilitating recruitment of a GAP to Gs or hindering the activation of $\mathrm{AC}$ by activated Gs. Irrespective of these mechanisms, the observation that $\mathrm{CRY}$ can modulate p-CREB levels also brings up potential roles for a CRY-CREB functional interaction in other functions of CREB. For example, the clock component $\mathrm{Per}$ genes also harbor CRE sites, and p-CREB has been shown to activate $P$ er transcription. This may underlie the simple feeding-fasting driven Per transcript oscillation in the absence of CRY proteins [10]. In the presence of a fully functional circadian clock and the CRY proteins, CRY fine-tunes p-CREB activity rhythms and enhances the robustness of the circadian clock and its downstream targets. Hence, the mechanism described here likely explains the synergistic interaction between the cell-autonomous circadian oscillator and feeding-fasting driven rhythms in sustaining robust genomewide transcriptional rhythms.

\section{References}

1 Zhang EE, Liu Y, Dentin R, et al. Cryptochrome mediates circadian regulation of cAMP signaling and hepatic gluconeogenesis. Nat Med 2010; 16:11521156.

2 Takahashi JS, Hong HK, Ko CH, et al. The genetics of mammalian circadian order and disorder: implications for physiology and disease. Nat Rev Genet 2008; 9:764-775.

3 Dibner C, Schibler U, Albrecht U. The mammalian circadian timing system: organization and coordination of central and peripheral clocks. Annu Rev Physiol 2010; 72:517-549.

4 Panda S, Antoch MP, Miller BH, et al. Coordinated transcription of key pathways in the mouse by the circadian clock. Cell 2002; 109:307-320.

5 Rudic RD, McNamara P, Curtis AM, et al. BMAL1 and CLOCK, two essential components of the circadian clock, are involved in glucose homeostasis. PLoS Biol 2004; 2:e377.

6 Turek FW, Joshu C, Kohsaka A, et al. Obesity and metabolic syndrome in circadian Clock mutant mice. Science 2005; 308:1043-1045.

7 Lamia KA, Storch KF, Weitz CJ. Physiological significance of a peripheral tissue circadian clock. Proc Natl Acad Sci USA 2008; 105:15172-15177.

8 Marcheva B, Ramsey KM, Buhr ED, et al. Disruption of the clock components CLOCK and BMAL1 leads to hypoinsulinaemia and diabetes. Nature 2010 ; 466:627-631.

9 Miller BH, McDearmon EL, Panda S, et al. Circadian and CLOCK-controlled regulation of the mouse transcriptome and cell proliferation. Proc Natl Acad Sci USA 2007; 104:3342-3347.

10 Vollmers C, Gill S, DiTacchio L, et al. Time of feeding and the intrinsic circadian clock drive rhythms in hepatic gene expression. Proc Natl Acad Sci USA 2009; 106:21453-21458.

11 Kornmann B, Schaad O, Bujard H, et al. System-driven and oscillator-dependent circadian transcription in mice with a conditionally active liver clock. 
PLoS Biol 2007; 5:e34.

12 Ross EM, Wilkie TM. GTPase-activat- ing proteins for heterotrimeric $G$ proteins: regulators of $\mathrm{G}$ protein signaling
(RGS) and RGS-like proteins. Annu Rev Biochem 2000; 69:795-827. 